\title{
Efficiently Making Secure Two-Party Computation Fair
}

\author{
Handan Kılınç ${ }^{1}$ and Alptekin Küpçü ${ }^{2}$ \\ 1 EPFL, Koç University \\ handan.kilinc@epfl.ch \\ ${ }^{2}$ Koç University \\ akupcu@ku.edu.tr
}

\begin{abstract}
Secure two-party computation cannot be fair against malicious adversaries, unless a trusted third party (TTP) or a gradual-release type super-constant round protocol is employed. Existing optimistic fair two-party computation protocols with constant rounds are either too costly to arbitrate (e.g., the TTP may need to re-do almost the whole computation), or require the use of electronic payments. Furthermore, most of the existing solutions were proven secure and fair via a partial simulation, which, we show, may lead to insecurity overall. We propose a new framework for fair and secure two-party computation that can be applied on top of any secure two party computation protocol based on Yao's garbled circuits and zero-knowledge proofs. We show that our fairness overhead is minimal, compared to all known existing work. Furthermore, our protocol is fair even in terms of the work performed by Alice and Bob. We also prove our protocol is fair and secure simultaneously, through one simulator, which guarantees that our fairness extensions do not leak any private information. Lastly, we ensure that the TTP never learns the inputs or outputs of the computation. Therefore, even if the TTP becomes malicious and causes unfairness by colluding with one party, the security of the underlying protocol is still preserved.
\end{abstract}

\section{Introduction}

In two-party computation (2PC), Alice and Bob intend to evaluate a shared function with their private inputs. The computation is called secure when the parties do not learn anything beyond what is revealed by the output of the computation. Yao [64] introduced the concept of secure $2 \mathrm{PC}$ and gave an efficient protocol; but this protocol is not secure against malicious parties who try to learn extra information from the computation by deviating from the protocol. Many solutions $[54,63,38,47]$ are suggested to strengthen Yao's protocol against malicious adversaries.

When one considers malicious adversaries, fairness is an important problem. A fair computation should guarantee that Alice learns the output of the function if and only if Bob learns. This problem occurs since in the protocol one party learns the output earlier than the other party; therefore (s)he can abort the protocol after learning the output, before the other party learns it.

There are two main methods of achieving fairness in 2PC: using gradual release $[58,42,59]$ or a trusted third party (TTP) $[13,45]$. The gradual release based protocols $[23,11,7]$ let the parties gradually (bit by bit, or piece by piece) and verifiably reveal the result. Malicious party will have one bit (or piece) advantage if the honest party starts to reveal the result first. Yet, if the malicious party has more computational power, he can abort the protocol earlier and learn the result via brute force, while the honest party cannot. In this case, fairness is not achieved. Another drawback is the necessity of many rounds.

The $\boldsymbol{T T P}$ approach employs a third party that is trusted by both Alice and Bob. A simple solution would be to give the inputs to the TTP, who computes the outputs and distributes fairly. In terms of efficiency and feasibility though, the TTP should be used in the optimistic model [4], where he gets involved in the protocol only when there is a dispute between Alice and Bob. It is very important to give the TTP the minimum possible workload because otherwise the system will have a bottleneck. Another important concern is privacy. In an optimistic solution, if there is no dispute, the TTP should not even know a computation took place, and 
even with a dispute, the TTP should never learn the inputs or outputs, or even identities. We achieve all these efficiency and privacy requirements on the TTP.

Another problem regarding fairness in secure two-party computation is the proof methodology. In previous works [42,13,59], fairness and security (with abort) were proven separately, only partially simulating the protocol (partial simulation). However, it is important to simulate everything together to ensure that the fairness solution does not leak any information beyond the original secure two-party computation requirement. Therefore, as in the security of the secure two-party computation, there should be ideal/real world simulation (see Section 2) that covers both fairness and security (full simulation). In other words, the simulator should learn the output in the real world only after it is guaranteed that both parties can learn the output in the real world to achieve ideal and real world indistinguishability of the outputs.

Our Contributions: The main achievement of this work is an efficient framework for making secure $2 \mathrm{PC}$ protocols fair, such that it guarantees fairness and security together, and can work on top of secure two party computation protocols extending Yao's garbled circuits to the malicious setting via zero-knowledge proofs (e.g., [38, 13, 29]). Note that the state-of-the-art optimistic fairness solution [13] is also based on zero-knowledge proofs.

- We use a simple-to-understand ideal world definition to achieve fairness and security together, and prove our protocol's security and fairness with full simulation which means proving security and fairness together.

- We show that proving security and fairness separately via only partial simulation is not necessarily secure (see Section 5).

- Our framework employs a trusted third party (TTP) for fairness, in the optimistic model. The TTP's load is very light: verification of signatures and commitments, and decryption only. If there is no dispute, the TTP does not even know a computation took place, and even with a dispute, the TTP never learns the inputs, outputs, or even identities of Alice and Bob. So, a semi-honest TTP is enough in our construction to achieve fairness.

- If the TTP becomes malicious (e.g., colludes with one of the parties), it does not violate the security of the underlying $2 \mathrm{PC}$ protocol; only the fairness property of the protocol is contravened.

- Our framework is also fair about the work done by Alice and Bob, since both of them perform the same steps in the protocol.

- The principles for fairness in our framework can be adopted by any $2 \mathrm{PC}$ protocol based on Yao's garbled circuits, employing zero knowledge proofs for the malicious setting, thereby achieving fairness with little overhead.

- We compare our framework with related fair secure two-party computation work and show that we achieve better efficiency and security.

\section{Related Works}

Cachin and Camenisch [13] present a state-of-the-art fair two-party computation protocol in the optimistic model. The protocol consists of two intertwined verifiable secure function evaluations. In the case of an unfair situation, the honest party interacts with the TTP. The job of the TTP can be as bad as almost repeating the whole computation, linear in the circuit size, creating a bottleneck in the system. Lindell [45] constructs a framework that can be adopted by any two-party functionality with the property that either both parties receive the output, or one party receives the output while the other receives a digitally-signed check (i.e., monetary compensation). However, one may argue that one party obtaining the output and the other obtaining the money may not always be considered fair, since we do not necessarily know how valuable the output would be before the evaluation. Kılınç and Küpçü [39] construct a fair multi-party computation (MPC) protocol in the optimistic model. While 2PC can be a special case of MPC, our solutions are optimized for the two-party case and hence are more efficient compared to applying their work to the two-party setting (e.g., they increase input and output sizes). 
Secure Two-Party Computation: Yao [64] presented a secure and efficient protocol for two-party computation in the semi-honest model, where the adversary follows the protocol's rules but he cannot learn any additional information from his view of the protocol. Since this protocol is not secure against malicious behavior, new methods were suggested based on proving parties' honesty via zero-knowledge proofs $[28,13,38]$ or via cut-and-choose techniques $[47,54,63,46]$.

Another problem in Yao's protocol is fairness. The general solutions are based on gradual release timed commitments $[58,42,59]$ or a trusted third party (TTP) $[13,45]$.

Gradual Release: Pinkas [58] presents a method where the evaluation of the garbled circuit is executed on the commitments of the garbled values rather than the original garbled values. It uses cut-and-choose to prevent malicious behavior of the constructor and blind signatures [16] for the verification of the evaluator's commitments. However, this protocol is expensive because of the gradual release timed commitments. Moreover, the majority circuit evaluation can reveal the constructor's input values, as shown by Kiraz and Schoenmakers [42], who improve Pinkas's construction by removing the majority circuit computation and the blind signatures, and using OR-proofs instead [21]. Yet, the other inefficiency problems remain. Similarly, Ruan et al. [59] use the Jarecki and Shmatikov construction [38], and instead of the proof of the correct garbled circuit, they employ the cut-and-choose technique. Gradual release in these protocols constitutes the weak part regarding the efficiency.

Security Definitions: Pinkas [58] protocol does not have proofs in the ideal-real world simulation paradigm. The importance of ideal-real world simulation is explained by Lindell and Pinkas [48]. The standard simulation definition [31] does not include fairness because of the impossibility result of fairness without honest majority [18]. Garay et al. [26] relax the notion of fairness and define a "commit-prove-fair-open" functionality to simulate gradual release to prove security and fairness together. Then, Kiraz and Schoenmakers [42] and Ruan et al. [59] use this functionality in their security proofs, but the output indistinguishabilty of the ideal and real worlds is not satisfied in their proofs because fairness is proven separately, using partial simulation. Therefore, none of these protocols has a full simulation proof in ideal-real world simulation paradigm that shows the protocol is both secure and fair. Cachin and Camenisch [13] give an ideal-real world definition that includes full simulation (fairness and security together) for the protocols that employ a TTP. This definition considers also malicious TTP but the definition does not use TTP in optimistic model since the universal trusted party in the ideal world contacts with the real world TTP in their ideal world definition. Interestingly, they do not prove their proposed protocol according to their definition. Lindell [45] defines a new ideal world definition that captures security and fairness as defined above (i.e., exchanging money in return is considered fair as well $[6,43])$ and proves fairness and security according to this definition. Kılınç and Küpçü [39] prove their fair and secure MPC protocol with full simulation. In Section 5, we show that proving fairness and security separately via partial simulation do not necessarily yield to fair and secure protocols. The security and fairness definition we use follows the same intuition as that of Canetti [15].

Relaxed Fairness: Because of the impossibility result on general fairness, alternative definitions arise as partial fairness $[26,5,34,30]$ and fairness in rational settings $[55,35,36,50$, $1,56]$.

Fairness for Specific Functionalities: Finally, there are very efficient constructions for specific functionalities $[33,9,24,2,3,19]$, but we are interested in computing general functionalities efficiently. We achieve this goal, fairly, in the malicious setting.

Definition 1 (Ideal World). It consists of the corrupted party $C$, the honest party $H$, and the universal trusted party $\mathfrak{U}$ (not the TTP). The ideal protocol is:

1. $\mathfrak{U}$ receives input $x$ or the message $\mathrm{ABORT}$ from $C$, and $y$ from $H$. If the inputs are invalid or $C$ sends the message ABORT, then $\mathfrak{U}$ sends $\perp$ to both of the parties and halts.

2. Otherwise $\mathfrak{U}$ computes $f(x, y)=\left(f_{c}(x, y), f_{h}(x, y)\right)$. Then, he sends $f_{c}(x, y)$ to $C$ and $f_{h}(x, y)$ to $H$. 
The outputs of the parties in an ideal execution between the honest party $H$ and an adversary $\mathcal{A}$ controlling $C$, where $\mathfrak{U}$ computes $f$, is denoted $\operatorname{IDEAL}_{f, \mathcal{A}(w)}(x, y, s)$ where $x, y$ are the respective inputs of $C$ and $H, w$ is an auxiliary input of $\mathcal{A}$, and $s$ is the security parameter.

The standard secure two-party ideal world definition $[48,31]$ lets the adversary $\mathcal{A}$ to ABORT after learning his output but before the honest party learns her output. Thus, proving protocols secure using the old definition would not meet the fairness requirements.

Definition 2 (Real World). The real world consists of, besides the parties, an adversary $\mathcal{A}$ that controls one of the parties, and the TTP who is involved in the protocol when there is unfair behavior. The pair of outputs of the honest party and the adversary $\mathcal{A}$ in the real execution of the protocol $\pi$, possibly employing the TTP, is denoted $\operatorname{REAL}_{\pi, \mathrm{TTP}, \mathcal{A}(w)}(x, y, s)$, where $x, y, w$ and $s$ are like above.

Note that $\mathfrak{U}$ and TTP are not related to each other. TTP is part of the real protocol to solve the fairness problem when it is necessary, but $\mathfrak{U}$ is not real.

Definition 3 (Fair and Secure Two-Party Computation). Let $\pi$ be a probabilistic polynomial time (PPT) protocol and let $f$ be a PPT two-party functionality. We say that $\pi$ computes $f$ fairly and securely if for every non-uniform PPT real world adversary $\mathcal{A}$ attacking $\pi$, there exists a non-uniform PPT ideal world adversary $S$ so that for every $x, y, w \in\{0,1\}^{*}$, the ideal and real world outputs are computationally indistinguishable:

$$
\left\{\operatorname{IDEAL}_{f, S(w)}(x, y, s)\right\}_{s \in \mathbb{N}} \equiv_{c}\left\{\operatorname{REAL}_{\pi, \operatorname{TTP}, \mathcal{A}(w)}(x, y, s)\right\}_{s \in \mathbb{N}}
$$

For optimistic protocols, to simulate the complete view of the adversary, the simulator also needs to simulate the behavior of the TTP for the adversary. This simulation also needs to be indistinguishable.

The closest such definition was given by Cachin and Camenisch [13]. Their definition's advantage is that it also considers misbehaving TTP, but their ideal world contacts the real world TTP, mixing both worlds. Thus, it does not fit the optimistic usage of TTP. We prefer to use the Definition 3, which is more intuitive and general (it can even include gradual release since it is not specific to only the protocols with TTP), to prove our proposed protocol in Section 4 because we use the TTP in the optimistic model and we assume that the TTP is semi-honest while proving the protocol.

Note that in our ideal world, the moment the adversary sends his input, $\mathfrak{U}$ computes the outputs and performs fair distribution. Thus, the adversary can either abort the protocol before any party learns anything useful, or cannot prevent fairness. This is represented in our proof with a simulator who learns the output only when it is guaranteed that both parties can learn the output. Also observe that, under this ideal world definition, if the simulator learns the output in the ideal world but the adversary aborts in the real world, that simulation would be distinguishable.

Suppose that Alice is malicious and $S$ simulates the behavior of honest Bob in the real world and the behavior of malicious Alice in the ideal world. Assume $S$ learns the output of Alice from $\mathfrak{U}$ in order to simulate the real protocol before it is guaranteed that in a real protocol both of the parties could receive their outputs. Further suppose that the adversarial Alice then aborts the protocol so that $S$ does not receive his output in the real world. Thus, in the real world the real Bob would have aborted, whereas the ideal Bob outputs the result of the computation. Clearly, the ideal and real worlds are distinguishable in this case. The proofs in $[59,42,13]$ unfortunately fall into this pitfall.

Definition 4. (Verifiable Escrow) An escrow is a ciphertext under the public key of the TTP. $A$ verifiable escrow [4, 14] enables the recipient to verify, using only the public key of TTP, that the plaintext satisfies some relation. A public non-malleable label can be attached to a verifiable escrow [62].

Communication Model: We do not need private and authenticated channels between the TTP and the parties. When there is dispute between the two parties, the TTP resolves 
the conflict atomically, which means the TTP interacts with either Alice or Bob at a given time, until that resolution is complete. We assume that the adversary cannot prevent the honest party from reaching the TTP eventually. We do not assume anything else about the communication model; our protocol's needs are minimal.

Notation: Alice and Bob will evaluate a function $f(x, y)=\left(f_{a}(x, y), f_{b}(x, y)\right)$, where Alice has an input $x$ and gets an output $f_{a}(x, y)$, and Bob has an input $y$ and gets an output $f_{b}(x, y)$, $f:\{0,1\}^{\ell} \times\{0,1\}^{\ell} \rightarrow\{0,1\}^{\ell} \times\{0,1\}^{\ell}$, where $\ell$ is a positive integer. For simplicity, we assume Alice and Bob have $\ell$-bit inputs and outputs each. Alice's input bits are $x=\left\{x_{1}, x_{2}, \ldots, x_{\ell}\right\}$ and Bob's input bits are $y=\left\{y_{1}, y_{2}, \ldots, y_{\ell}\right\}$. They use a $2 \mathrm{PC}$ protocol $\Gamma$ for the secure computation.

We use $\mathfrak{C}$ to represent circuit. $\mathfrak{C}_{a}$ outputs the Alice's output and $\mathfrak{C}_{b}$ outputs Bob's output. Similarly, the garbled circuit that is generated by Alice is $\mathrm{GC}_{a}$ and the one generated by Bob is $\mathrm{GC}_{b}$. We use apostrophe (') for the values that are generated by Bob. When we say Alice's input wires, it means that Alice provides the input for these wires. Similarly, Alice's output wires correspond to Alice's output. Bob's input and output wires have the matching meaning. An Input Gate is a gate that has an input wire of Alice or Bob. Similarly, an Output Gate is a gate that has a wire of Alice's or Bob's output.

$E_{k}$ shows an encryption with the key $k$. Therefore, $E_{k_{1}} E_{k_{2}}\left(m_{1}, m_{2}\right)$ means that $m_{1}$ and $m_{2}$ are both encrypted by the two keys $k_{1}$ and $k_{2}$.

Any commitments that have efficient zero knowledge proofs can be used in this framework. To exemplify the protocol we notate commitments as in Fujisaki-Okamoto commitments [25, 22] and Pedersen commitments [57].

\section{Our Solution}

Failed Approaches and Major Issues: It looks like adding fairness to a $2 \mathrm{PC}$ protocol based on gabled circuits and zero knowledge using TTP does not need a lot of work. However, if we care efficiency of the protocol and resolution protocols with the TTP, it is challenging. Consider a very simple solution regarding constructor C, evaluator E, and the TTP. Assume that $\mathrm{C}$ constructs the circuit such that the output is not revealed directly, but instead the output of the circuit is an encrypted version of the real output, and $\mathrm{C}$ knows the key. Thus, after evaluation, $\mathrm{E}$ will learn this encrypted output, and $\mathrm{C}$ and $\mathrm{E}$ need to perform a fair exchange of this encrypted output and the key. This approach increases the circuit size, obviously. Besides, when a dispute occurs and E goes to the TTP for resolution, she cannot efficiently prove to the TTP that she evaluated C's garbled circuit correctly. Indeed, in the solution of Cachin and Camenisch [13], the resolution may require work proportional to the circuit size.

Alternatively, instead of encrypting the output, $\mathrm{C}$ constructs a garbled circuit where the outputs are encoded with some random values (like an encryption but without increasing the circuit size) in a secret table. So, in the end of the circuit evaluation, E learns some random values such that their corresponding bits are only known by C. Then, they can fairly exchange the table and the output. However, it can be hard to ensure that $\mathrm{E}$ sends the correct table and construct proper resolution protocols with TTP.

Because of these issues, we employ the dual-constructor methodology [52,53], where both $\mathrm{C}$ and $\mathrm{E}$ construct circuits that output random numbers.

Our Solution: We show how to efficiently add fairness to any zero knowledge based secure 2PC protocol $\Gamma$ using our framework. The key points are:

- Alice and Bob employ dual garbling technique [52], where Alice and Bob both act as the constructor and the evaluator, with almost equal responsibilities. The circuit constructed by Alice only outputs Alice's output and the circuit constructed by Bob outputs Bob's output.

The garbled circuit is prepared as the underlying protocol $\Gamma$ with minor differences in the construction of the input and output gates. The modification on the input gates allow us to check input equality between the two circuits. Modifications on the output gates are to hide the actual output. 
- Alice and Bob exchange the garbled circuits and evaluate each others' circuits. In the end of evaluation, Alice learns the output labels of Bob and Bob learns the output labels of Alice, both in a hidden way. Therefore, they need to exchange the outputs fairly after this point.

- Before fair exchange, they execute input equality test protocol to see if both of them used the same inputs for the both circuits. It is ok to abort if the test fails, because they test for input equality, not output.

- If the equality test is successful, they verifiably escrow the other party's output labels. This is essentially a guarantee for the other party that if this party does not send the output labels later on, (s)he can contact the TTP to get them.

- Now, they exchange output labels so that each party can individually translate them back to the actual outputs, since they come from circuits that they themselves created. If there is a dispute about the fairness, they go to the TTP for the resolution.

Overview of the resolution protocols is the following:

Alice/Bob Resolve: We describe the resolution for Bob, though it is completely symmetric for Alice. Remember that Bob is equipped with a verifiable escrow. But, for the TTP to decrypt it for him, Bob must prove that he acted properly. He provides output labels of Alice, and proves that they are evaluated from Alice's garbled circuit. If so, the TTP provides the decryption for Bob, who can use it to translate back to his output bits.

Alice Abort: Alice may try to abort the protocol and block resolution attempts with the TTP, should she not receive Bob's verifiable escrow. When she contacts the TTP, if Bob has resolved before, she obtains her output labels from the TTP. Otherwise, the TTP marks the protocol as aborted, and would deny any resolution attempt by Alice or Bob.

Note that the TTP only sees random output labels, but not their translation tables. Furthermore, since each circuit only evaluates to one party's output, even if the TTP colludes with the malicious party and provides the other party's output labels, those are still meaningless without the corresponding bits. Thus, a malicious TTP may only break fairness, but not security.

Why Target Zero-Knowledge Proof based Garbled Circuit Protocols? We claimed that our framework can be applied on top of any zero-knowledge proof based garbled circuit protocols. There are two reasons for this:

1. As explained above, parties commit to output labels, for enabling efficient resolutions with the TTP (one of the major problems in previous work). They must prove to each other that they committed to the correct labels as in the garbled circuits. If the underlying protocol, for example, encrypts the garbled tables using AES, then such a proof cannot be efficiently done (without cut-and-choose), whereas if the underlying encryption scheme is numbertheoretic (such as simplified Camenisch-Shoup [14,38]), then using sigma protocols [21], the correctness proofs may be done very efficiently.

2. Item 1 above leaves out the cut-and-choose way of proving. The problem is that, if cut-andchoose is employed, then there will be multiple circuits, rather than one. In our solution, parties create verifiable escrows, and the TTP may need to decrypt them. Verifiable escrow is a primitive that inherently uses zero-knowledge proofs. It is unclear how to combine the verifiable escrow idea with cut-and-choose, where multiple circuits exist, especially when the TTP needs to be able to verify and decrypt them.

In essence, one may think of our solution as a framework that can be applied on top of $2 \mathrm{PC}$ schemes that employ a single circuit, and use number-theoretic constructions (of encryption) for efficiency.

\section{Making Secure 2PC Fair (Full Protocol)}

Notation: Alice and Bob will evaluate a function $f(x, y)=\left(f_{a}(x, y), f_{b}(x, y)\right)$, where Alice has an input $x$ and gets an output $f_{a}(x, y)$, and Bob has an input $y$ and gets an output $f_{b}(x, y)$, $f:\{0,1\}^{\ell} \times\{0,1\}^{\ell} \rightarrow\{0,1\}^{\ell} \times\{0,1\}^{\ell}$, where $\ell$ is a positive integer. For simplicity, we assume 
Alice

$\left(x, \mathfrak{C}, P K_{\mathrm{TTP}}, i d\right)$

pick $\left(s k_{a}, v k_{a}\right)$
Bob

$\left(y, \mathfrak{C}, P K_{\mathrm{TTP}}, i d\right)$

pick $\left(s k_{b}, v k_{b}\right)$

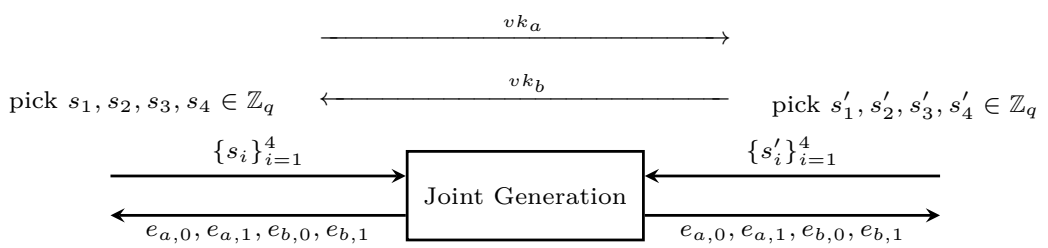

For input gates

pick $u_{1}, u_{2}, \ldots, u_{\ell} \in \mathbb{Z}_{q}$

$$
\begin{gathered}
\left\{m_{a_{i}, 0}=e_{a, 0}^{u_{i}}\right\}_{i=1}^{\ell},\left\{m_{a_{i}, 1}=e_{a, 1}^{u_{i}}\right\}_{i=1}^{\ell} \\
\left\{m_{b_{i}, 0}=e_{b, 0}^{u_{i}}\right\}_{i=1}^{\ell},\left\{m_{b_{i}, 1}=e_{b, 1}^{u_{i}}\right\}_{i=1}^{\ell} \\
\text { pick }\left\{r_{i, 0}, r_{i, 1}\right\}_{i=1}^{\ell} \in \mathbb{Z}_{q} \\
\left\{\mathrm{D}_{b_{i}, 0}=m_{b_{i}, 0} h^{r_{i, 0}}, \mathrm{D}_{b_{i}, 1}=m_{b_{i}, 1} h^{r_{i, 1}}\right\}_{i=1}^{\ell} \\
\text { For output gates }
\end{gathered}
$$

pick $\left\{p_{i}=\left(\delta_{i}, \epsilon_{i}\right)\right\}_{i=1}^{4 \ell}, \mathcal{S}_{a}=\left\{\mathrm{S}_{i}=g^{\delta_{i}} h^{\epsilon_{i}}\right\}_{i=1}^{4 \ell}$

$$
\begin{gathered}
\text { For input gates } \\
\text { pick } u_{1}^{\prime}, u_{2}^{\prime}, \ldots, u_{\ell}^{\prime} \in \mathbb{Z}_{q} \\
\left\{m_{a_{i}, 0}^{\prime}=e_{a, 0}^{u_{i}^{\prime}}\right\}_{i=1}^{\ell},\left\{m_{a_{i}, 1}^{\prime}=e_{a, 1}^{u_{i}^{\prime}}\right\}_{i=1}^{\ell} \\
\left\{m_{b_{i}, 0}^{\prime}=e_{b, 0}^{u_{i}^{\prime}}\right\}_{i=1}^{\ell},\left\{m_{b_{i}, 1}^{\prime}=e_{b, 1}^{u_{i}^{\prime}}\right\}_{i=1}^{\ell} \\
\operatorname{pick}\left\{r_{i, 0}^{\prime}, r_{i, 1}^{\prime}\right\}_{i=1}^{\ell} \in \mathbb{Z}_{q} \\
\left\{\mathrm{D}_{a_{i}, 0}^{\prime}=m_{a_{i}, 0}^{\prime} h^{r_{i, 0}^{\prime}}, \mathrm{D}_{a_{i}, 1}^{\prime}=m_{a_{i}, 1}^{\prime} h^{r_{i, 1}^{\prime}}\right\}_{i=1}^{\ell} \\
\text { For output gates } \\
\text { pick }\left\{p_{i}^{\prime}=\left(\delta_{i}^{\prime}, \epsilon_{i}^{\prime}\right)\right\}_{i=1}^{4 \ell}, \mathcal{S}_{b}=\left\{\mathrm{S}_{i}^{\prime}=g^{\delta_{i}^{\prime}} h^{\epsilon_{i}^{\prime}}\right\}_{i=1}^{4 \ell}
\end{gathered}
$$

S2PC phase

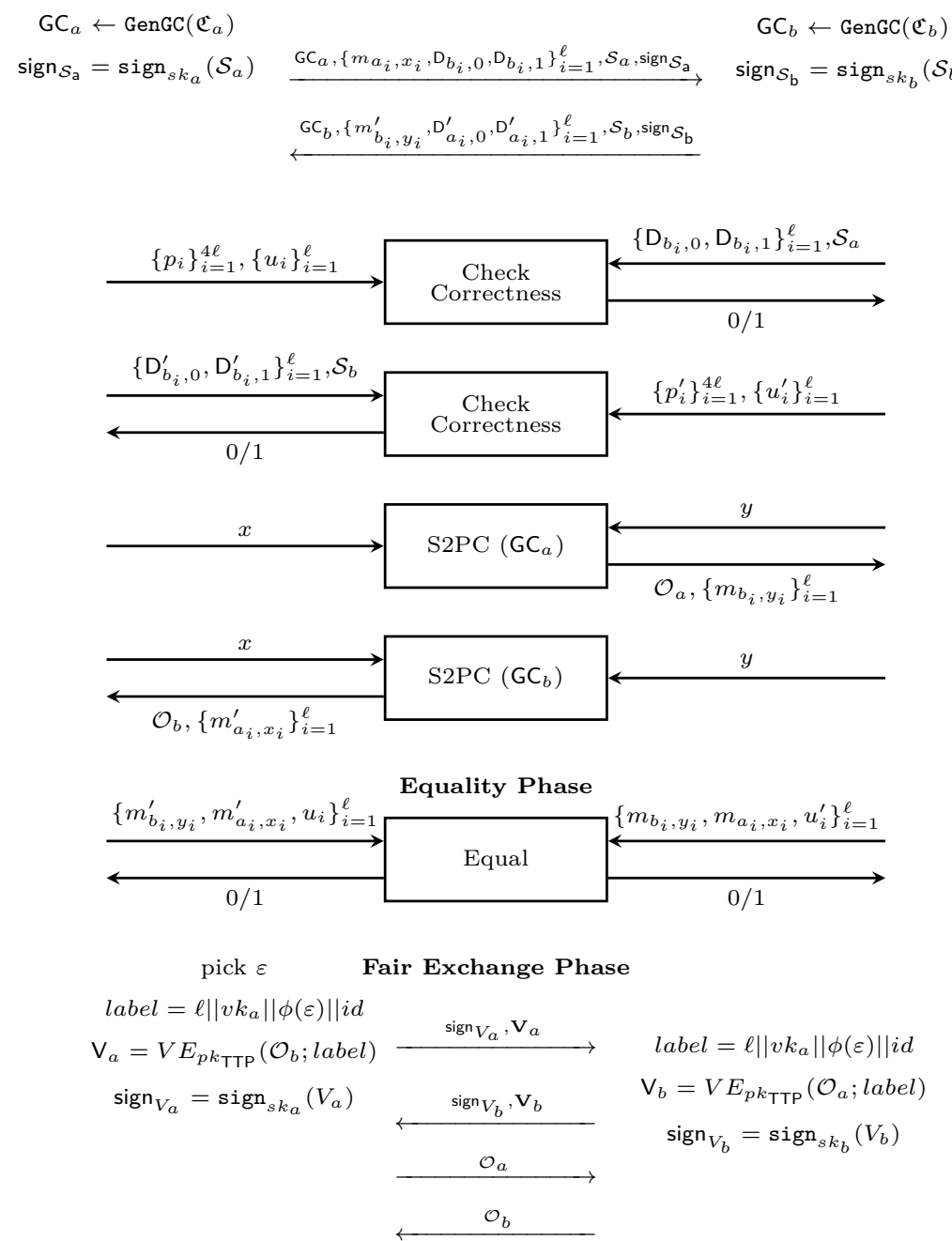

Fig. 1. Our framework to make a S2PC protocol fair. GenGC generates garbled circuit. 
Alice and Bob have $\ell$-bit inputs and outputs each. Alice's input bits are $x=\left\{x_{1}, x_{2}, \ldots, x_{\ell}\right\}$ and Bob's input bits are $y=\left\{y_{1}, y_{2}, \ldots, y_{\ell}\right\}$. They use a $2 \mathrm{PC}$ protocol $\Gamma$ for the secure computation.

We use $\mathfrak{C}$ to represent circuit. $\mathfrak{C}_{a}$ outputs the Alice's output and $\mathfrak{C}_{b}$ outputs Bob's output. Similarly, the garbled circuit that is generated by Alice is $\mathrm{GC}_{a}$ and the one generated by Bob is $\mathrm{GC}_{b}$. We use apostrophe (') for the values that are generated by Bob. When we say Alice's input wires, it means that Alice provides the input for these wires. Similarly, Alice's output wires correspond to Alice's output. Bob's input and output wires have the matching meaning. An Input Gate is a gate that has an input wire of Alice or Bob. Similarly, an Output Gate is a gate that has a wire of Alice's or Bob's output.

$E_{k}$ shows an encryption with the key $k$. Therefore, $E_{k_{1}} E_{k_{2}}\left(m_{1}, m_{2}\right)$ means that $m_{1}$ and $m_{2}$ are both encrypted by the two keys $k_{1}$ and $k_{2}$.

Any commitments that have efficient zero knowledge proofs can be used in this framework. To exemplify the protocol we notate commitments as in Fujisaki-Okamoto commitments [25, 22] and Pedersen commitments [57].

Table 1. The review of the random numbers used for fairness in our framework.

\begin{tabular}{|c|c|l|}
\hline Name & Form & Relation \\
\hline Equality-Test Constants & $e=g^{\rho}$ & $\begin{array}{l}\text { There are four kinds of them, where each repre- } \\
\text { sents 0 or 1 and right or left. }\end{array}$ \\
\hline Input-Gate Randoms & $u$ & $\begin{array}{l}\text { Each input gate has them. They are private; just } \\
\text { known by the constructors. }\end{array}$ \\
\hline Equality-Test Numbers & $m=e^{u}$ & $\begin{array}{l}\text { For each input-gate random } u \text {, there are four } \\
\text { kinds of them, where each represents 0 or 1 and } \\
\text { right or left according to } e .\end{array}$ \\
\hline Output Labels & $(\delta, \varepsilon)$ & $\begin{array}{l}\text { They are randomly chosen pairs, each represent- } \\
\text { ing a row of the garbled output gates. }\end{array}$ \\
\hline
\end{tabular}

We give a review of the random numbers that are used for fairness in Table 1. The protocol steps are described in detail below (and in Figure 1).

The TTP generates the group $\mathcal{G}_{1}$ that is used in $\Gamma$ and picks generators $g, h \in \mathcal{G}_{1}$, secret and public key pair $s k_{T T P}, p k_{T T P}$ for the verifiable escrow scheme. Additionally, he chooses a cyclic group $\mathcal{G}_{2}$ whose order is a large prime $q$ and randomly selects its generators $g_{0}, g_{1}, g_{2}$ (for the equality test). He also picks a one-way function $\phi()$. Then, he announces his public key $P K_{\mathrm{TTP}}=\left[p k_{T T P},\left(\mathcal{G}_{1}, g, h\right),\left(\mathcal{G}_{2}, q, g_{0}, g_{1}, g_{2}\right), \phi()\right]$.

Both Alice and Bob know $P K_{\text {TTP }}$ and agree on a circuit $\mathfrak{C}$ that computes $f(x, y)$ and the protocol identifier $i d$ before the protocol begins.

Preparation Phase:

1. Alice and Bob generate private-public key pairs $\left(s k_{a}, v k_{a}\right)$ and $\left(s k_{b}, v k_{b}\right)$, respectively, for an unforgeable signature scheme. They exchange the signature verification keys $v k_{a}$ and $v k_{b}$.

They jointly generate four equality-test constants $e_{a, 0}, e_{a, 1}, e_{b, 0}$ and $e_{b, 1}$ as described [40]. Equality test constants represent 0 and 1 for the left $(a)$ and the right $(b)$ wires of the input gates.

2. Alice and Bob separately generate the random numbers and commitments for the input and the output gates as shown in Figure 1.

The computations of Alice and Bob for each input gate $i$ are the following: inputgate numbers $\left(u_{i}\right.$ resp. $\left.u_{i}^{\prime}\right)$, the equality-test numbers $\left(\left\{t \in\{0,1\}, z \in\{a, b\}: m_{z_{i}, t}=\right.\right.$ $\left.e_{z, t}^{u_{i}}\right\}$ resp. $\left.\left\{t \in\{0,1\}, z \in\{a, b\}: m_{z_{i}, t}^{\prime}=e_{z, t}^{u_{i}^{\prime}}\right\}\right)$, and the commitments $\left(\left\{t \in\{0,1\}: \mathrm{D}_{b_{i}, t}=\right.\right.$ $\left.m_{b_{i}, t} h^{r_{i, t}}\right\}$ resp. $\left.\left\{t \in\{0,1\}: \mathrm{D}_{a_{i}, t}^{\prime}=m_{a_{i}, t}^{\prime} h^{r_{i, t}^{\prime}}\right\}\right)$. They are used in the input equality test to show the same inputs are used for both garbled circuits.

They generate output labels $\left(\left(\delta_{j}, \epsilon_{j}\right)\right.$ resp. $\left.\left(\delta_{j}^{\prime}, \epsilon_{j}^{\prime}\right)\right)$ for each row of garbled-output gate $j$ and their commitments $\left(\mathrm{S}_{j}\right.$ resp. $\left.\mathrm{S}_{j}^{\prime}\right)$ for the output gates. The sets of the commitments are $\mathcal{S}_{a}=\left\{\mathrm{S}_{j}\right\}$ resp. $\mathcal{S}_{b}=\left\{\mathrm{S}_{j}^{\prime}\right\}$. The output labels are as unique identifiers for the rows of the constructor's garbled-output gates. Only the constructor knows which row they represent, which means only the constructor knows which output bit they correspond to. This makes sure that the evaluator cannot learn the output directly.

S2PC Phase: 
1. [Garbled Circuits:] Alice and Bob construct their garbled circuits by following the rules of the underlying $\Gamma$ protocol with little differences on the garbled tables of the input and the output gates.

Table 2. The garbled Input and Output Gate for an OR gate constructed by Alice. Encryption scheme is same as the underlying protocol $\Gamma$.

\begin{tabular}{|c|c|c|}
\hline Row & Garbled Input Gate & Garbled Output Gate \\
\hline 00 & $E_{k_{a_{i}, 0}} E_{k_{b_{i}, 0}}\left(r_{i, 0}, k_{0}\right)$ & $E_{k_{a, 0}} E_{k_{b, 0}}\left(\delta_{j}, \varepsilon_{j}\right)$ \\
\hline 01 & $E_{k_{a_{i}, 0}} E_{k_{b_{i}, 1}}\left(r_{i, 1}, k_{1}\right)$ & $E_{k_{a, 0}} E_{k_{b, 1}}\left(\delta_{j+1}, \varepsilon_{j+1}\right)$ \\
\hline 10 & $E_{k_{a_{i}, 1}} E_{k_{b_{i}, 0}}\left(r_{i, 0}, k_{1}\right)$ & $E_{k_{a, 1}} E_{k_{b, 0}}\left(\delta_{j+2}, \varepsilon_{j+2}\right)$ \\
\hline 11 & $E_{k_{a_{i}, 1}} E_{k_{b_{i}, 1}}\left(r_{i, 1}, k_{1}\right)$ & $E_{k_{a, 1}} E_{k_{b, 1}}\left(\delta_{j+3}, \varepsilon_{j+3}\right)$ \\
\hline
\end{tabular}

Input Gates: The difference is that each garbled-table row of an input gate $i$ includes one more encryption besides the encryption of the output key. It is the encryption of either $r_{i, 0}^{\prime}$ or $r_{i, 1}^{\prime}$ representing the input of 0 and 1 for the wire of Alice in $\mathrm{GC}_{b}$ and either $r_{i, 0}$, or $r_{i, 1}$ representing the input of 0 and 1 for the wire of the Bob in $\mathrm{GC}_{a}$. See Table 2 for the details.

Remark: Alice and Bob just encrypt the partial decommitments of $\mathrm{D}_{b_{i}, 0}, \mathrm{D}_{b_{i}, 1}$ and $\mathrm{D}_{a_{i}, t}^{\prime} \mathrm{D}_{a_{i}, 1}^{\prime}$, respectively because they only need to learn equality-test numbers ( $m$ values) that represent their input bits. They do not want to reveal input-gate numbers ( $u$ values) since it causes the evaluator to learn the constructor's input.

Remark: Note that there can be just one input wire of a gate (e.g., NOT gate for negation). In this case, there will be two equality-test numbers which represent 0 and 1 for this gate. Alternatively, they can agree to construct a circuit using only NAND gates [13].

Output Gates: Each row of the garbled output gate includes the encryption of corresponding output labels instead of encryption of real output bits (see Table 2). This is to hide the actual output from the evaluator.

2. [Exchange:] They exchange the constructed garbled tables along with the commitments, the signature of all commitments of the output labels $\left(\operatorname{sign}_{\mathcal{S}_{a}}\right.$ resp. $\left.\operatorname{sign}_{\mathcal{S}_{b}}\right)$ and equality-test numbers that represents their input bits as in Figure 1.

3. [Check Correctness:] They prove to each other that they performed the input and the output gates' construction honestly, via efficient zero-knowledge proofs :

- Proof of Input Gates to prove that the garbled input gates contain the correct decommitment values. This is basically done in three steps:

Firstly, prover proves that (s)he knows the decommitmets of all commitments denoted by $\mathrm{D}$ [14]. Secondly, prover proves that each commitment pair $\mathrm{D}_{z, 0}$ and $\mathrm{D}_{z, 1}$ commits the same value under the different bases $e_{z, 0}$ and $e_{z, 1}$ respectively. If the prover is Alice then $z=b_{i}$, if the prover is Bob then $z=a_{i}$. Lastly, the prover proves that each input-garbled table includes the double-encryption of partial decommitment of $\mathrm{D}_{z, 0}$ and $\mathrm{D}_{z, 1}$.

- Proof of Output Gates to prove that the garbled output gates encrypt the committed output labels.

If there is a problem in the proofs, they abort. Otherwise, they continue.

4. [S2PC:] Alice and Bob execute $\Gamma$, and evaluate the garbled circuit they were given. While executing $\Gamma$, Alice and Bob prove that they correctly construct their garbled circuits that evaluate $f$ by zero-knowledge proofs described in the protocol $\Gamma$. If all zero-knowledge proofs are verified, at the end of the evaluation, Alice learns the set $\mathcal{O}_{b}$ representing $f_{b}$, Bob learns the set $\mathcal{O}_{a}$ representing $f_{a}$, each including $\ell$ output labels. Besides, each party learns the set that includes equality-test numbers that represents her/his input (from the decryption of input-garbled gates). Otherwise, they abort.

Equality Phase: This phase is necessary to test whether or not Alice and Bob used the same input bits for both circuit evaluations. We use unfair version of equality test by Boudot et al. [9]; the unfair version is sufficient for our purpose. 
Alice and Bob want to check, if $x_{i}^{*}=x_{i}$ and $y_{i}^{*}=y_{i}$ for the encryptions $E_{k_{a_{i}, x_{i}}^{\prime}}\left(E_{k_{b_{i}, y_{i}}^{\prime}}\left(k^{\prime}\right)\right)$ and $E_{k_{a_{i}, x_{i}^{*}}}\left(E_{k_{b_{i}, y_{i}^{*}}}(k)\right)$ in each garbled input gate $i$, such that the first one was decrypted by Alice and the second one was decrypted by Bob. For this purpose, Alice and Bob will use the equality-test numbers $\left\{m_{z_{i}, t}, m_{z_{i}, t}^{\prime}\right\}_{z \in\{a, b\}, t \in\{0,1\}}$.

Assume Alice decrypted a row for an input gate $i$ and learned equality-test numbers $m_{a_{i}, x_{i}}^{\prime}$ and she knows $m_{b_{i}, y_{i}}^{\prime}$ since Bob sent his equality-test numbers that represents his input in the exchange step of the S2PC phase. Also assume Bob decrypted the corresponding garbled gate and similarly learned $m_{b_{i}, y_{i}^{*}}$ and he knows $m_{a_{i}, x_{i}^{*}}$ since Alice sent it in the exchange step of the S2PC phase. If they both used consistent input bits for both $G C_{a}$ and $G C_{b}$, then we expect to see that the following equation is satisfied:

$$
\left(m_{a_{i}, x_{i}}^{\prime} m_{b_{i}, y_{i}}^{\prime}\right)^{u_{i}}=\left(m_{a_{i} x_{i}^{*}} m_{b_{i}, y_{i}^{*}}\right)^{u_{i}^{\prime}}
$$

The left hand side of the equation (1) is composed of values Alice knows since she learned $m^{\prime}$ values and generated $u_{i}$ herself. Similarly, the right hand side values are known by Bob since he learned $m$ values and generated $u_{i}^{\prime}$ himself. This equality should hold if $x_{i}^{*}=x_{i}$ and $y_{i}^{*}=y_{i}$ since $m_{a_{i}, x_{i}^{*}}^{\prime}=e_{a, x_{i}^{*}}^{u_{i}^{\prime}}, m_{b_{i}, y_{i}^{*}}^{\prime}=e_{b, y_{i}^{*}}^{u_{i}^{\prime}}$ and $m_{a_{i}, x_{i}}=e_{a, x_{i}}^{u_{i}}, m_{b_{i}, y_{i}}=e_{b, y_{i}}^{u_{i}}$.

After computing their side locally in equation (1) for each input gate, they concatenate the results in order to hash them, where the output range of the hash function is $\mathbb{Z}_{q}$. Then Alice and Bob execute Proof of Equality protocol in [9] with the hashes.

If the equality test succeeds, they continue with the next phase.

Remark: Remember that the constructor did not prove that (s)he added equality-test numbers to the correct row of the encryption table. Suppose that the constructor encrypted the equality-test number that represents 0 where the evaluator's encryption key represents 1 . In this case, it is sure that the equality test will fail, but the important point is that the constructor cannot understand which row is decrypted by the evaluator, and thus does not learn any information because he cannot cheat just in one row. If he cheats in one row, he has to change one of the other rows as well, as otherwise he fails the "Proof of Input Gates". Thus, even if the equality test fails, the evaluator might have decrypted any one of the four possibilities for the gate, and thus might have used any input bit. This also means that the equality test can be simulated, and hence reveals nothing about the input.

Note that there are some techniques to check input equality in the literature as in $[47,42$, $49,61,52,53]$ but they are based on cut-and-choose. Since the underlying protocol $\Gamma$ does not use cut-and-choose to guarantee the security, the equality test we used is more suitable here.

Fair Exchange Phase: In this phase, Alice and Bob exchange the outputs. Remember that the outputs are indeed randomized, and only the constructor knows their meaning. Thus, if they do not perform this fair exchange, no party learns any information about the real output (unless they resolve with the TTP, in which case they both learn their outputs).

1. Alice first picks a value $\omega$ from the domain of the one way-function $\phi$ and computes $\phi(\omega)$. Next, she creates a verifiable escrow $\mathrm{V}_{a}$ including $\mathcal{O}_{b}$ with non-malleable label $\left(\ell\left\|v k_{a}\right\| \phi(\varepsilon) \| i d\right)$ as in Figure 1. Finally, she signs $\mathrm{V}_{a}$ with $s k_{a}$ and sends the signature $\operatorname{sign}_{V_{a}}$ and $\mathrm{V}_{a}$.

With the verifiable escrow, she proves that there are $\ell$ different decommitments in the escrow that correspond to $\ell$ of the commitments in $\mathcal{S}_{b}[37,20,10]$. Since Alice can just decrypt one row for every gate and so she only has one pair of keys for each gate, this proof shows that Alice decrypted Bob's garbled output tables correctly, and the verifiable escrow has the evaluation result of $\mathrm{GC}_{b}$. If $\mathrm{V}_{a}$ or sign $V_{a}$ fails to verify, or if the label is not correct, then Bob aborts. Otherwise, Bob continues with the next step.

Remark: $\omega$ is used in the Alice Abort protocol with the TTP to prevent Bob from claiming to be Alice and aborting after Bob Resolve. Since only Alice knows $\varepsilon$ that is a pre-image of $\phi(\varepsilon)$, Bob cannot convince the TTP.

2. Bob creates a verifiable escrow $\bigvee_{b}$ including $\mathcal{O}_{a}$ with non-malleable label the same as Alice created. He signs $\mathrm{V}_{b}$ with $s k_{b}$ and sends the signature $\operatorname{sign}_{V_{b}}$ and $\mathrm{V}_{b}$.

With the verifiable escrow, he proves that there are $\ell$ different decommitments in the escrow that correspond to $\ell$ of the commitments in $\mathcal{S}_{a}[37,20,10]$. If $\mathrm{V}_{a}$ or $\operatorname{sign}_{V_{a}}$ fails to verify, or 
if the label is not correct, then Alice runs "Alice Abort" protocol with the TTP. Otherwise, Alice continues with the next step.

3. Alice sends $\mathcal{O}_{b}$ to Bob.

4. Bob checks if the output labels in $\mathcal{O}_{b}$ are correct. The output labels are correct if $\ell$ of them are the pairs that are generated by Bob. If they are correct, then he sends $\mathcal{O}_{a}$. If at least one of the output labels is not correct, then he does "Bob Resolve" with the TTP.

5. Alice checks if the output labels in $\mathcal{O}_{a}$ are correct. If they are not correct, then she does "Alice Resolve" with the TTP. Otherwise the protocol ends.

Alice and Bob Resolve (See Figure 2): We explain Bob Resolve below. Alice Resolve is the same where the verifiable escrow, the signatures and $\mathcal{O}$ are Bob's values.

Bob contacts with the TTP and sends the values $\mathrm{V}_{a}, \operatorname{sign}_{V_{a}}, \mathcal{S}_{a}, \operatorname{sign}_{\mathcal{S}_{a}}, \mathcal{O}_{a}$. He sends sign $\mathcal{S}_{a}$ to prove that $\mathcal{S}_{a}$ is generated by the same party who generates $\mathrm{V}_{a}$. The TTP checks if all signatures are correct and the decommitments in $\mathcal{O}_{a}$ correspond to $\ell$ of the commitments in $\mathcal{S}_{a}$. If there is no problem, then the TTP decrypts $\mathrm{V}_{a}$ with $s k_{T T P}$ and sends the values inside $\mathrm{V}_{a}$ to Bob. Since Bob knows the meaning of the output labels of the garbled circuit he constructed, he effectively learns his output. The TTP remembers Alice's output $\mathcal{O}_{a}$, given and proven by Bob, in his database.

Alice Abort (See Figure 3): When Alice contacts the TTP for abort, she sends $\mathrm{V}_{a}$ and $\operatorname{sign}_{a}$, together with $\varepsilon$. The TTP checks that the signature is valid and $\phi(\varepsilon)$ matches the label of $\mathrm{V}_{a}$. If Bob did resolve before, the TTP sends $\mathcal{O}_{a}$ as in Figure 3 so that Alice can also learn her output. Otherwise, the protocol is aborted and the TTP will not honor resolution requests for this exchange.

Remark that Alice and Bob do not re-do the zero-knowledge proofs in the S2PC phase to the TTP because sign $V_{V_{a}}$ and sign $V_{b}$ show that both Alice and Bob execute everything correctly until the end of Equality Phase.

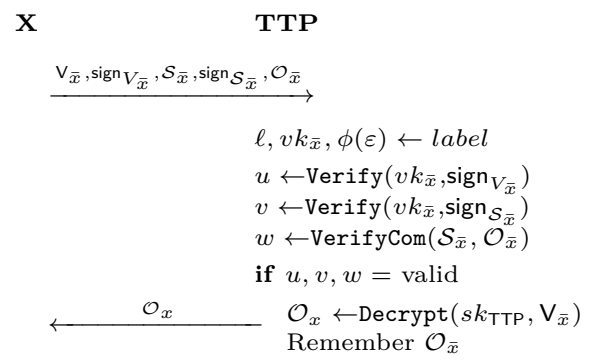

Fig. 2. $X$ Resolve where $X \in\{$ Alice,Bob $\}$. If $\mathrm{X}$ is Alice, $\bar{x}$ is $b$, otherwise $\bar{x}$ is $a$.

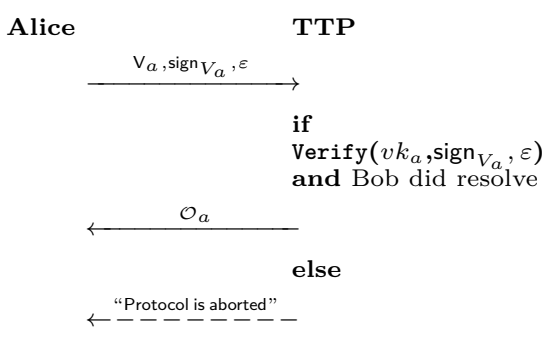

Fig. 3. Alice Abort

Theorem 1. Let $f:\{0,1\}^{*} \times\{0,1\}^{*} \rightarrow\{0,1\}^{*} \times\{0,1\}^{*}$ be any probabilistic polynomial time (PPT) two-party functionality. The protocol above for computing $f$ is secure and fair according to Definition 3, assuming that the TTP is semi-honest, the subprotocols that are stated in the protocol are all secure (sound and zero-knowledge), all commitments are hiding and binding [25, 22], the signature scheme used is unforgeable [32], and the $\Gamma$ is a 2PC protocol secure against malicious adversaries based on Yao's garbled circuits and zero knowledge proofs.

\section{Security against Malicious Alice:}

Lemma 1. Under the assumptions in Theorem 1, our protocol is secure and fair against malicious Alice $\mathcal{A}$.

Proof. We construct a simulator $S_{B}$ that interacts with Alice in the real world as Bob, and with $\mathfrak{U}$ in the ideal world as Alice:

1. Preparation Phase: $S_{B}$ generates the same values as in the preparation phase. 
2. S2PC Phase: $S_{B}$ constructs the garbled circuit the same as the simulator of $\Gamma$. For the input and output gates, it follows the same process as in the framework. Then, $\mathcal{A}$ and $S_{B}$ exchange the garbled circuits and commitments.

$\mathcal{A}$ performs check correctness phase. Here, $S_{B}$ learns all input gate numbers $\left\{u_{i}\right\}_{i=1}^{\ell}$ from the proof of input gates and all output labels $\left\{\left(\delta_{i}, \epsilon_{i}\right)\right\}_{i=1}^{4 \ell}$ from the proof of output gates using soundness extractors. If $\mathcal{A}$ 's proofs are not valid, then $S_{B}$ sends message ABORT to $\mathfrak{U}$ and stops. Otherwise, they continue with the evaluation of circuits.

$S_{B}$ learns the input of $\mathcal{A}$ which is $x=\left(x_{1}, x_{2}, \ldots, x_{\ell}\right)$ by using the simulator of $\Gamma$.

3. Equality Phase: $S_{B}$ acts as the random oracle, so when $\mathcal{A}$ asks for the hash of her equality test input, $S_{B}$ answers her randomly and sees equality-test numbers that corresponds $\mathcal{A}$ 's input, which consist of $m^{\prime}$ values. Since these were generated by $S_{B}$, he can check if they are the expected ones because $S_{B}$ knows his own inputs and Alice's inputs for $S_{B}$ 's garbled circuit, so he knows which row $\mathcal{A}$ has to decrypt.

In addition, $S_{B}$ checks if $\mathcal{A}$ used the same input in $\mathrm{GC}_{a}$. He knows which row $\mathcal{A}$ should have decrypted, as explained above. He learned the input-gate numbers $\left\{u_{i}\right\}_{i=1}^{\ell}$ in the previous step. So $S_{B}$ can learn which equality-test constants of the row that he decrypted are used, and hence he can learn that $\mathcal{A}$ used which input in $\mathrm{GC}_{a}$. Finally, $S_{B}$ can check if the inputs that $\mathcal{A}$ used in both circuits are equal.

They begin the equality test protocol. If the proofs of $\mathcal{A}$ are not valid or the input values are not the expected ones, then $S_{B}$ sends ABorT message to $\mathfrak{U}$. $S_{B}$ simulates all zero-knowledge proof of knowledge protocols in the equality test, similarly as in [9]. If the test was supposed to return true, he simulates that way, otherwise he simulates with another random value whose hash is random. The probability that the adversary queries the random oracle at this random value is negligible. If the result is false, then he sends ABORT message to $\mathfrak{U}$ and the simulation ends.

Remark: Our security theorem intentionally does not mention the random oracle model. As is specified above and in [9], the equality test and the use of the hash function here necessitates the use of the random oracle model. Yet, one may convert this part to be secure in the standard model, with a loss of efficiency. First, all zero-knowledge proofs of knowledge must be done interactively. Second, instead of hashing the values and performing only a single equality test, the values must be compared independently, using $2 l$ (sequential) equality tests. Performing these equality tests independently does not harm security, since one may not cheat in the other party's input, but can only cheat with own input, and therefore learns nothing from the (in)equality.

4. Fair Exchange Phase: If the equality test is successful, then $\mathcal{A}$ should send the signature $\operatorname{sign}_{V_{a}}$ and $\bigvee_{a}$. If $\mathcal{A}$ sends them and verification fails, then $S_{B}$ sends ABORT message to $\mathfrak{U}$. Otherwise, the simulation continues with the next step.

Otherwise, it is certain that both parties can obtain the output, because honest real Bob could have obtained his output using the Bob Resolve, and thus there is no more need for aborting. Hence, $S_{B}$ hands Alice's input $x$ to $\mathfrak{U}$ and $\mathfrak{U}$ sends $f_{a}(x, y)$ to the simulator.

If $\mathcal{A}$ does "Alice Abort" in any point above: If $S_{B}$ went to $\mathfrak{U}$ before, then $S_{B}$ simulates the TTP and gives output labels corresponding to the $\mathcal{A}$ 's output $f_{a}(x, y)$ to $\mathcal{A}$ and if $S_{B}$ have not been contacted with $\mathfrak{U}$ yet, then then $S_{B}$ sends ABORT message to $\mathfrak{U}$ and the real protocol is aborted as well.

Remember that $S_{B}$ extracted all output labels of $\mathcal{A}$ from the Proof of Output Gates. In addition, he learned which bit the row represents from the Proof of Garbled Construction. If no "Alice Abort" is executed, then he picks output labels corresponding to the $\mathcal{A}$ 's output $f_{a}(x, y)$ and sends all to $\mathcal{A}$.

5. Resolutions: $S_{B}$ waits for the response from $\mathcal{A}$. If $\mathcal{A}$ does not respond or sends invalid output labels corresponding to his output, then he simulates Bob Resolve by decrypting $\mathrm{V}_{a}$. Later on, if $\mathcal{A}$ contacts the TTP for the resolution, $S_{B}$ simulates the TTP using the output labels.

6. $S_{B}$ outputs whatever $\mathcal{A}$ outputs. 
Claim. The view of $\mathcal{A}$ in his interaction with the simulator $S_{B}$ is indistinguishable from the view in his interaction with real Bob and real TTP.

Proof. We need to check the behaviors of the $S_{B}$ that are different from Bob's behavior in the protocol, since these can affect the distribution.

- The construction garbled circuit of $S_{B}$ is indistinguishable than the real one thanks to the simulator in protocol $\Gamma$.

- $S_{B}$ did not commit to the real equality-test numbers as real Bob would do. Since the commitment scheme is hiding, the fake commitments are indistinguishable from the real ones. In addition, $S_{B}$ simulates the zero-knowledge proofs in the protocol. Their indistinguishably comes from the zero-knowledge simulatability.

- The simulator only aborts when the real Bob would abort. Thus, the abort actions are indistinguishable as well.

- The simulator can perfectly simulate the TTP as well, since he generated its keys.

We emphasize one more time that a simulation proof for a secure and fair two-party computation protocol needs to make sure that the simulator learns the output only when it is guaranteed that the other party can also obtain the output, and not before that point. In real world, it is sure for honest Bob that he will learn the functionality output after Alice sends the correct verifiable escrow, because even if Alice does not send the output to him in the fair exchange phase, Bob can learn them from the TTP via Bob Resolve. For this reason $S_{B}$ sends input $x$ to $\mathfrak{U}$ after receiving the valid verifiable escrow. After that Bob and $S_{B}$ learn their outputs in the ideal world. As mentioned, Bob learns his output certainly in the end of the protocol in the real world after this point. Since Bob is honest, $\mathcal{A}$ learns her output too, in the real world.

Overall, we proved that the joint distribution of $\mathcal{A}$ and the honest Bob's output in the real execution is indistinguishable from the joint distribution of $S_{B}$ and the honest Bob's output in the ideal model.

Therefore, combined with Claim 4, the proof of Lemma 1 is complete.

\section{Security against Malicious Bob:}

Lemma 2. Under the assumptions in Theorem 1, our protocol is secure and fair against malicious Bob.

Proof. The proof is similar to that of Lemma 1. We construct a simulator $S_{A}$ that interacts as Alice with the adversary $\mathcal{B}$ that controls Bob in the real world, and as Bob with the $\mathfrak{U}$ in the ideal world. $S_{A}$ simulates the protocol as following:

1. Up to Fair Exchange Phase: All simulation actions are the same as the simulator $S_{B}$ above until the end of equality test.

2. Fair Exchange Phase: $S_{A}$ prepares verifiable escrow (if the equality test was indeed successful) and the signature $\operatorname{sign}_{V_{a}}$ and. Since $S_{A}$ does not know $f_{a}(x, y)$, she adds random values in the escrow. $S_{A}$ simulates the proof by using verifiable escrow simulator [14].

$S_{A}$ waits for the verifiable escrow of $\mathcal{B}$. If $\mathcal{B}$ sends $\mathrm{V}_{b}$ with $\operatorname{sign}_{V_{b}}$ and they are correct, we are guaranteed that the other party can obtain the output. So, the protocol will not be aborted. $S_{A}$ sends $y$, which he learned via the $\Gamma$ simulator, to $\mathfrak{U}$, and gets $f_{b}(x, y)$ from $\mathfrak{U}$. This also means that $\mathfrak{U}$ sends $f_{a}(x, y)$ to Alice in the ideal world. Since $S_{A}$ learned all decommitments of output labels of Bob from Proof of Output Gates, $S_{A}$ prepares the output labels of $\mathcal{B}$ according to $f_{b}(x, y)$ and sends them to $\mathcal{B}$.

If $\mathrm{V}_{b}$ or sign ${ }_{b}$ are not valid, $S_{A}$ sends ABORT to $\mathfrak{U}$. If $\mathcal{B}$ tries to do "Bob Resolve" then $S_{B}$ simulates the TTP and sends abort message to $\mathcal{B}$.

3. Resolutions:

$S_{A}$ waits for the response from $\mathcal{A}$. If $\mathcal{B}$ does not respond or sends invalid output labels corresponding to her output, then she simulates Alice Resolve by decrypting the verifiable escrow. Later on, if $\mathcal{B}$ contacts the TTP for resolution, $S_{B}$ simulates the TTP using the output labels in the previous step. 
4. $S_{A}$ outputs whatever $\mathcal{B}$ outputs.

Claim. The view of $\mathcal{B}$ in his interaction with the simulator $S_{A}$ is indistinguishable from the view in his interaction with real Alice and real TTP.

Proof. Honest Alice learns the output of the function if malicious Bob sends it in the fair exchange phase, or if Bob successfully performs Bob Resolve in the real world. For this reason, the simulator $S_{A}$ learns the output from $\mathfrak{U}$ in the ideal world exactly at these points. The abort cases also follow the real world.

As the rest of the simulation is similar to the case in Claim 4 , the joint distribution of $\mathcal{B}$ and honest Alice's output in the real execution is indistinguishable from the joint distribution of $S_{A}$ and honest Alice's output in the ideal model.

This completes the proof of Theorem 1 .

\subsection{TTP Analysis}

As we claim, a semi-honest TTP is sufficient in our protocol because the TTP only learns output labels where their meaning is only known by the circuit constructors (Alice or Bob), and a signature. In addition, (s)he does not receive anything else related to the input of Alice or Bob. Furthermore, the signature key can be specific to each circuit, and thus we do not require a public key infrastructure, and it does not give away the parties' identity. ${ }^{3}$ Therefore, if the TTP follows the protocol but also tries to learn extra information about the parties (input or output), (s)he cannot succeed.

Even if the TTP is malicious, (s)he can only break the fairness property of the protocol. A malicious TTP can collude with Alice or Bob. If Alice or Bob colludes with the TTP, they cannot break the correctness property because in the honest Bob case, he cannot receive wrong output since Alice can only learn one output label for each output gate (learning only one output label per gate), so she can use only the learned output labels, which means TTP cannot give different ones (because (s)he only knows those that Alice provides). Thus, the TTP cannot break the correctness property. A similar argument shows that an honest Alice cannot receive a wrong output.

\section{Proving Security and Fairness Together}

In this section we show the importance of proving with full simulation according to Definition 3. First, we define what we mean by partial simulation more formally and then we give contrived versions of several protocols $([41,59,13])$ including ours that are obviously insecure, but can be proven fair and secure with partial simulation while it cannot be proven fair and secure with full simulation.

Definition 5 (Partial Simulation). Let $f, h, g$ be the PPT functionalities where $f=g \circ h$, $h(x, y)=\left(h_{b}, h_{a}\right)$ and $g\left(h_{b}, h_{a}\right)=\left(f_{a}, f_{b}\right)$ and let $\pi_{f}, \pi_{h}, \pi_{g}$ be the PPT protocols to compute $f, h, g$, respectively where the first input and output of a functionality correspond to one party (Alice) and the second input and output of a functionality correspond to other party (Bob). The partial simulation paradigm says that $\pi_{f}$ computes $f$ fairly and securely if there exists a PPT protocol $\pi_{h}$ that is secure under simulation with abort [27] and there exists a PPT protocol $\pi_{g}$ which achieves fairness [4, 43].

Almost all previous works (See Table 3) prove their fairness and security with partial simulation: prove security with the unfair simulation paradigm (with abort) (corresponding to

\footnotetext{
${ }^{3}$ The discussion about the usage of unique identifiers for the TTP to identify different evaluations between some Alice and some Bob (with anonymity and unlinkability guarantees), thereby hiding identities exist in previous work $[4,43]$. Therefore, we do not complicate our presentation with such issues.
} 
proving $\pi_{h}$ to be a secure $2 \mathrm{PC}$ protocol with abort), and argue fairness (of the $\pi_{g}$ part, either using TTP or gradual release) separately. This is risky. Consider the following three contrived protocols where Alice and Bob want to compute functionality $f=\left(f_{a}, f_{b}\right)$ fairly and securely:

- A modification on our protocol is that the TTP gives Alice's output along with the input of Bob whenever Alice contacts for resolution or abort, if Bob have done "Bob Resolve" before (honest Bob is required to provide his input to the TTP in "Bob Resolve"). Here, $h=\left(h_{a}, h_{b}\right)$ is a functionality where $h_{a}=\mathcal{O}_{a}$ and $h_{b}=\mathcal{O}_{b}\left(\pi_{h}\right.$ is our protocol until the fair exchange phase, where parties only obtain random output labels), and $g$ is a functionality where $g\left(\mathcal{O}_{a}, \mathcal{O}_{b}\right)=\left(f_{x}, f_{y}\right)\left(\pi_{g}\right.$ is the fair exchange phase of our protocol with new "Alice Abort" and "Alice Resolve".). It is very easy to simulate $\pi_{h}$ with abort, since parties essentially learn nothing. Also, it is easy to argue about fairness of this $\pi_{g}$ without simulation, since at the end of resolutions, either both parties obtain their outputs or no one learns anything useful.

- The protocol which is the same as Cachin and Camenisch's protocol [13] where the only difference is that the TTP gives the other parties' inputs to the party in the resolution protocols (with similar reasoning as above).

- The modified versions of Kiraz and Schoenmakers [42] or Ruan et. al [59] protocols where the only difference is Alice sends her input to Bob and vice versa at the end of the gradual release.

In $[42,59]$ partial simulation is provided only until the beginning of the gradual release phase, then fairness is argued via the fairness of the gradual release. Similarly, in [13] the partial simulation is provided for a functionality computation, then the fairness is discussed based on the parties' and TTP's behaviors. Using the same type of reasoning, their and our contrived versions can be proven fair and secure via partial simulation, and fairness can be argued since at the end of the gradual release or TTP resolutions, either both parties obtain their outputs or no one does. But, it is clear that the contrived protocols leak the inputs to the other party, becoming insecure. Observe that they can never be fully simulated, because the simulator will not have access to the honest party's input and so it cannot provide indistinguishability of ideal and real worlds.

Consequently, it is risky to argue fairness separate from the ideal/real world simulation. We do not claim that previous protocols $[13,42,59]$ have security problems, but we want to emphasize that the partial simulation technique does not cover all security aspects of a protocol and should not be preferred anymore. Therefore, they should be proven with the full simulation technique.

Importance of the Timing of the Simulator contacting the Universal Trusted Party: The proofs of the protocols $[59,42,13]$ are also problematic since the simulator learns the output of the computation from $\mathfrak{U}$ before it is guaranteed that the other party can also obtain the output. This behavior of the simulator violates the indistinguishibility of the ideal and real worlds because if the simulator does not receive his/her output in the real world while the parties already obtained the outputs in the ideal world, then the outputs in ideal and real worlds are distinguishable, and the simulation fails. Therefore, the simulator must obtain the output from the universal trusted party in the ideal world, only after it is guaranteed that both parties can obtain the output in the real world.

\section{Performance}

To add fairness to the underlying $\Gamma$ protocol, each party needs to compute extra $4 \ell$ exponentiations, $6 \ell$ commitments, 2 signatures, $8 \ell$ verifiable encryptions containing 2 values each, $4 \ell$ proofs of knowledge, and a verifiable escrow containing $\ell$ values. Among all these computation, the most time consuming one is verifiable encryption. According to [51], a verifiable encryption with two values takes almost 318 milliseconds (ms) and with one value takes 257 ms. For the verifiable escrow containing $\ell$ values, we have at most $0.25 \ell$ seconds ${ }^{4}$. Therefore, we can

\footnotetext{
${ }^{4}$ The computation time of a verifiable escrow with $\ell$ values is less than $0.25 \ell$ seconds since it does not increase linearly, but we estimate an upper bound here.
} 
conclude that each party spends at most 3.05 $\ell$ seconds for the extra computations. Hence, our framework is independent from the circuit size and we can conclude that our overhead is $O(\ell)$. Our overhead compared to the time that is spend for the $\Gamma$ protocol is very small because $\ell$ is expected to be much much less than the circuit size. For example, if $\Gamma$ computes AES encryption, $\ell=128$ and the circuit size is around 30000 gates. In this case, each party spends extra 6.5 minutes which is minimal compared to 30000 circuit evaluation (in zero-knowledge based garbled circuits).

If a resolution protocol is executed, TTP verifies one signature, checks $\ell$ commitments and decrypts one verifiable escrow. The most time consuming part for TTP is the decryption of the verifiable escrow, which takes time around $5 \ell \mathrm{ms}[51]$.

Our protocol adds extra 10 messages with the joint generation, the equality test, and the fair exchange, while gradual release based solutions $[58,42,59]$ require much more number of rounds (based on the security parameter).

When we examine our network overhead, we have extra 100.6 $\ell$ KB (kilobyte) from verifiable encryptions and $18.8 \ell \mathrm{KB}$ from verifiable escrow [51]. ${ }^{5}$

\section{Conclusion}

Table 3 presents a comparison with the most related works.

Table 3. Comparison of our protocol with previous works. CC denotes cut-and-choose, ZK denotes efficient zero-knowledge proofs of knowledge, GR denotes gradual release, OFE denotes efficient optimistic fair exchange, superscript $I$ denotes inefficient TTP, superscript $P$ denotes necessity of using a payment system, NS denotes no ideal-real simulation proof given, PS indicates partial simulation proof, and finally FS indicates full simulation proof including fairness. A check mark $\checkmark$ is put for easily identifying better techniques.

\begin{tabular}{|l|l|l|l|c|c|r|}
\hline & {$[58]$} & {$[59]$} & {$[42]$} & {$[45]$} & {$[13]$} & Ours \\
\hline Malicious Behavior & CC & CC & CC & CC $/$ ZK & ZK & ZK \\
\hline Fairness & GR & GR & GR & OFE $^{P}$ & OFE $^{I}$ & OFE $\checkmark$ \\
\hline Proof Technique & NS & PS & PS & FS $\checkmark$ & PS & FS $\checkmark$ \\
\hline
\end{tabular}

$\checkmark$ All our overhead (TTP, Alice, Bob) are dependent only on the input and output size, and independent of the circuit size, in contrast to [13].

$\checkmark$ We require a constant number of rounds for fairness, contrary to gradual release based solutions $[58,42,59]$.

$\checkmark$ We do not necessitate a payment framework. Our fairness definition is that either both parties obtain the output, or no one does, as opposed to [45].

$\checkmark$ Even if the TTP becomes malicious and colludes with one participant, he cannot violate the security of the protocol. On the other hand, in [45], while the Bank cannot violate 2PC security, it can maliciously deal with the balances, possibly causing a lot of headache.

$\checkmark$ Finally, our protocol is proven secure in the ideal/real simulation paradigm (not in [58]) with output indistinguishability (not in $[42,59,13]$ ), and by proving fairness and security simultaneously via a full simulation proof (none except [45]).

\section{Acknowledgements}

The authors acknowledge the support of TÜBİTAK, the Scientific and Technological Research Council of Turkey, under project number 111E019, and European Union COST Action IC1306.

\footnotetext{
${ }^{5}$ We do not consider other messages here, since they are neglectable compared to verifiable encryption and escrow.
} 


\section{References}

1. I. Abraham, D. Dolev, R. Gonen, and J. Halpern. Distributed computing meets game theory: robust mechanisms for rational secret sharing and multiparty computation. In Proceedings of the twenty-fifth annual ACM symposium on Principles of distributed computing.

2. G. Asharov. Towards characterizing complete fairness in secure two-party computation. In Theory of Cryptography, pages 291-316. Springer, 2014.

3. G. Asharov, A. Beimel, N. Makriyannis, and E. Omri. Complete characterization of fairness in secure two-party computation of boolean functions. In Theory of Cryptography, pages 199-228. Springer, 2015

4. N. Asokan, V. Shoup, and M. Waidner. Optimistic fair exchange of digital signatures. IEEE Journal on Selected Areas in Communications, pages 591-610, 2000.

5. D. Beaver and S. Goldwasser. Multiparty computation with faulty majority. In CRYPTO, 1990.

6. M. Belenkiy, M. Chase, C. Erway, J. Jannotti, A. Küpçü, A. Lysyanskaya, and E. Rachlin. Making P2P accountable without losing privacy. In ACM WPES, 2007.

7. D. Boneh and M. Naor. Timed commitments (extended abstract). In CRYPTO, 2000.

8. F. Boudot. Efficient proofs that a committed number lies in an interval. In EUROCRYPT, 2000.

9. F. Boudot, B. Schoenmakers, and J. Traoré. A fair and efficient solution to the socialist millionaires' problem. Discrete Applied Mathematics, (1-2):23-36, 2001.

10. E. Bresson and J. Stern. Proofs of knowledge for non-monotone discrete-log formulae and applications. In $I S C, 2002$.

11. E. F. Brickell, D. Chaum, I. Damgård, and J. v. d. Graaf. Gradual and verifiable release of a secret. In CRYPTO, 1987.

12. Brownie cashlib cryptographic library. http://github.com/brownie/cashlib.

13. C. Cachin and J. Camenisch. Optimistic fair secure computation. In CRYPTO, 2000.

14. J. Camenisch and V. Shoup. Practical verifiable encryption and decryption of discrete logarithms. In CRYPTO, 2003.

15. R. Canetti. Security and composition of multiparty cryptographic protocols. Journal of Cryptology, 13:143-202, 2000.

16. D. Chaum. Blind signatures for untraceable payments. In CRYPTO, 1982.

17. D. Chaum and T. P. Pedersen. Wallet databases with observers. In CRYPTO, 1993.

18. R. Cleve. Limits on the security of coin flips when half the processors are faulty. In STOC, 1986.

19. R. Cohen and Y. Lindell. Fairness versus guaranteed output delivery in secure multiparty computation. In Advances in Cryptology-ASIACRYPT 2014, pages 466-485. Springer, 2014.

20. R. Cramer, I. Damgård, and B. Schoenmakers. Proofs of partial knowledge and simplified design of witness hiding protocols. In CRYPTO, 1994.

21. I. Damgård. On sigma protocols. http://www.daimi.au.dk/ ivan/Sigma.pdf.

22. I. Damgard and E. Fujisaki. A statistically-hiding integer commitment scheme based on groups with hidden order. In ASIACRYPT, 2002.

23. I. B. Damgård. Practical and provably secure release of a secret and exchange of signatures. Journal of Cryptology, 8:201-222, 1995.

24. C. Dong, L. Chen, J. Camenisch, and G. Russello. Fair private set intersection with a semi-trusted arbiter. In DBSec, 2013.

25. E. Fujisaki and T. Okamoto. Statistical zero knowledge protocols to prove modular polynomial relations. In CRYPTO, 1997.

26. J. A. Garay, P. MacKenzie, M. Prabhakaran, and K. Yang. Resource fairness and composability of cryptographic protocols. Journal of cryptology, 2011.

27. O. Goldreich. Foundations of Cryptography: Volume 2, Basic Applications. Cambridge University Press, New York, NY, USA, 2004.

28. O. Goldreich, S. Micali, and A. Wigderson. How to play any mental game. In STOC, 1987.

29. O. Goldreich, S. Micali, and A. Wigderson. Proofs that yield nothing but their validity or all languages in $\mathrm{np}$ have zero-knowledge proof systems. Journal of ACM, 38:728, 1991.

30. S. Goldwasser and L. Levin. Fair computation of general functions in presence of immoral majority. In CRYPT0. 1991

31. S. Goldwasser and Y. Lindell. Secure computation without agreement. In Distributed Computing. Springer, 2002.

32. S. Goldwasser, S. Micali, and R. Rivest. A digital signature scheme secure against adaptive chosen-message attacks. SIAM Journal on Computing, 17:281-308, Apr. 1988.

33. S. D. Gordon, C. Hazay, J. Katz, and Y. Lindell. Complete fairness in secure two-party computation. Journal of ACM, 58, 2011. 
34. S. D. Gordon and J. Katz. Partial fairness in secure two-party computation. Journal of cryptology, 2012.

35. A. Groce and J. Katz. Fair computation with rational players. In EUROCRYPT. 2012.

36. J. Halpern and V. Teague. Rational secret sharing and multiparty computation. In Proceedings of the thirty-sixth annual ACM symposium on Theory of computing, 2004.

37. R. Henry and I. Goldberg. Batch proofs of partial knowledge. In ACNS, 2013.

38. S. Jarecki and V. Shmatikov. Efficient two-party secure computation on committed inputs. In EUROCRYPT, 2007.

39. H. Kılınç and A. Küpçü. Optimally efficient multi-party fair exchange and fair secure multi-party computation. In CT-RSA 2015.

40. H. Kılınç and A. Küpçü. Efficiently making secure two-party computation fair. Cryptology ePrint Archive, Report 2014/896.

41. M. S. Kiraz and B. Schoenmakers. A protocol issue for the malicious case of yaoâÁŹs garbled circuit construction. In In Proceedings of 27th Symposium on Information Theory in the Benelux, 2006.

42. M. S. Kiraz and B. Schoenmakers. An efficient protocol for fair secure two-party computation. In $C T-R S A, 2008$.

43. A. Küpçü and A. Lysyanskaya. Usable optimistic fair exchange. Computer Networks, 56:50-63, 2012.

44. A. Küpçü. Efficient Cryptography for the Next Generation Secure Cloud: Protocols, Proofs, and Implementation. Lambert Academic Publishing, 2010.

45. A. Y. Lindell. Legally-enforceable fairness in secure two-party computation. In $C T-R S A, 2008$.

46. Y. Lindell. Fast cut-and-choose based protocols for malicious and covert adversaries. In CRYPTO, 2013.

47. Y. Lindell and B. Pinkas. An efficient protocol for secure two-party computation in the presence of malicious adversaries. In EUROCRYPT, 2007.

48. Y. Lindell and B. Pinkas. Secure multiparty computation for privacy-preserving data mining. Journal of Privacy and Confidentiality, 1, 2009.

49. Y. Lindell and B. Pinkas. Secure two-party computation via cut-and-choose oblivious transfer. Journal of cryptology, pages 680-722, 2012.

50. A. Lysyanskaya and N. Triandopoulos. Rationality and adversarial behavior in multi-party computation. In CRYPTO. 2006.

51. S. Meiklejohn, C. C. Erway, A. Küpçü, T. Hinkle, and A. Lysyanskaya. Zkpdl: A language-based system for efficient zero-knowledge proofs and electronic cash. In USENIX Security Symposium, 2010.

52. P. Mohassel and M. Franklin. Efficiency tradeoffs for malicious two-party computation. In $P K C$, 2006.

53. P. Mohassel and B. Riva. Garbled circuits checking garbled circuits: More efficient and secure two-party computation. In CRYPTO. 2013.

54. J. B. Nielsen and C. Orlandi. Lego for two-party secure computation. In $T C C, 2009$.

55. S. J. Ong, D. C. Parkes, A. Rosen, and S. Vadhan. Fairness with an honest minority and a rational majority. In Theory of Cryptography. 2009.

56. S. J. Ong, D. C. Parkes, A. Rosen, and S. Vadhan. Fairness with an honest minority and a rational majority. In Theory of Cryptography. 2009.

57. T. P. Pedersen. Non-interactive and information-theoretic secure verifiable secret sharing. In CRYPTO, 1992.

58. B. Pinkas. Fair secure two-party computation. In EUROCRYPT, 2003.

59. O. Ruan, J. Chen, J. Zhou, Y. Cui, and M. Zhang. An efficient fair uc-secure protocol for two-party computation. Security and Communication Networks, 2013.

60. C.-P. Schnorr. Efficient signature generation by smart cards. Journal of Cryptology, pages 161-174, 1991.

61. C.-h. Shen et al. Two-output secure computation with malicious adversaries. In EUROCRYPT. 2011.

62. V. Shoup and R. Gennaro. Securing threshold cryptosystems against chosen ciphertext attack. In EUROCRYPT, 1998.

63. D. P. Woodruff. Revisiting the efficiency of malicious two-party computation. In EUROCRYPT, 2007.

64. A. C. Yao. Protocols for secure computations. In FOCS, 1982. 


\section{A Sub Protocols}

\section{A.1 Joint Generation}

For the joint generation, Bob first commits to four random elements from $\mathbb{Z}_{n}$ and proves the knowledge of the committed values and their ranges $[12,8]$. Then, Alice chooses four random elements from the same range and sends them to Bob. Finally, Bob decommits. Then they both calculate the multiplication of pairs of these numbers, in $\mathbb{Z}_{n}$, and so jointly agree on random numbers $\rho_{a, 0}, \rho_{a, 1}, \rho_{b, 0}, \rho_{b, 1}$. In the end, they calculate equality test constants where $\left\{e_{z, t}=g^{\rho_{z, t}}\right\}_{z \in\{a, b\}, t \in\{0,1\}}$.

\section{A.2 Check Correctness}

We present the subprotocols from Alice's viewpoint as the prover. Bob's proofs are symmetric.

Proof of Output Gates:

Inputs: Common inputs are the garbled tables of gate $g$ that contains encryptions $E_{00}, E_{01}, E_{10}, E_{11}$, along with the commitments $\mathrm{S}_{i}^{A}, \ldots, \mathrm{S}_{i+3}^{A}$ of the output labels of $g$. Prover's private inputs are all decommitments. Prover performs following:

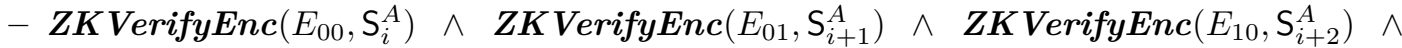
$\boldsymbol{Z} \boldsymbol{K} \operatorname{Verify} \boldsymbol{E n c}\left(E_{11}, \mathrm{~S}_{i+3}\right)$, where $\boldsymbol{Z K} \boldsymbol{V e r i f y} \boldsymbol{E n c}(E, S)$ proves that the encryption $E$ contains the decommitment of $\mathrm{S}$.

\section{Proof of Correct Input Gates:}

Inputs: Common inputs are the commitments $\mathrm{D}_{w_{b}, 0}^{A}, \mathrm{D}_{w_{b}, 1}^{A}$ and the garbled input gate table that has encryptions $E_{00}, E_{01}, E_{10}, E_{11}$. Prover knows the decommitments. Prover performs the following (details in [38] or appendix of [44]):

- According to prover's side, he performs one of the proofs below. If she is from the left side (in our protocol it is Alice's side) then she performs ZKVerifyEncRight, otherwise he

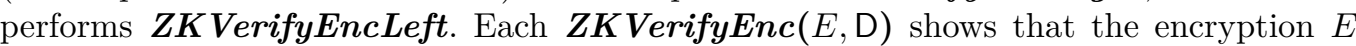
contains the decommitment of the commitment D. This proof can be done as in [14].

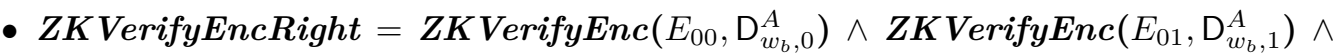

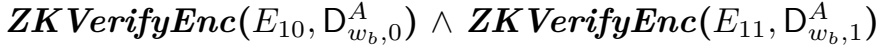

- $Z K$ VerifyEncLeft $=Z K$ VerifyEnc $\left(E_{00}, \mathrm{D}_{w_{b}, 0}^{A}\right) \wedge Z K \operatorname{VerifyEnc}\left(E_{01}, \mathrm{D}_{w_{b}, 0}^{A}\right) \wedge$

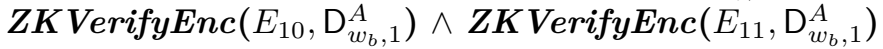

$-\boldsymbol{Z} \boldsymbol{K} \boldsymbol{C o m \boldsymbol { D }} \boldsymbol{L}\left(\mathrm{D}_{w_{b}, 0}^{A}\right) \wedge \boldsymbol{Z} \boldsymbol{K} \boldsymbol{C o m} \boldsymbol{D} \boldsymbol{L}\left(\mathrm{D}_{w_{b}, 1}^{A}\right)$ proving knowledge of the decommitments of these commitments.

- $\boldsymbol{Z} \boldsymbol{K} \boldsymbol{E} \boldsymbol{q} \boldsymbol{C o m} \boldsymbol{D} \boldsymbol{L}\left(\mathrm{D}_{w_{b}, 0}^{A}, \mathrm{D}_{w_{b}, 1}^{A}\right)$ showing that the committed messages are equal for both commitments (under different bases, $e_{b, 0}$ and $e_{b, 1}$ ).

\section{A.3 Equality Test}

The adapted protocol of [9] is the following:

- Alice and Bob generate a cyclic abelian group $G$, which has a large prime order $q$, with generators $g_{0}, g_{1}, g_{2}, g_{3}$. Then, they compute the corresponding values of their input bits (for equality test) in $\mathbb{Z}_{q}$. In our case, these are the hashed values of all left (right) hand sides of equation 1. Say Alice's value is $\theta_{a}$ and Bob's is $\theta_{b}$.

- Alice chooses a random $x_{a} \in \mathbb{Z}_{q}$ and computes $g_{a}=g_{1}^{x_{a}}$ and similarly Bob chooses a random $x_{b} \in \mathbb{Z}_{q}$ and computes $g_{b}=g_{1}^{x_{b}}$. Then, they send these values to each other and calculate $g_{a b}=g_{a}^{x_{b}}=g_{b}^{x_{a}}$. In addition, they prove knowledge of $x_{a}$ and $x_{b}$ using Schnorr's protocol [60].

- Alice selects $a \in_{R} \mathbb{Z}_{q}$, calculates $\left(P_{a}, Q_{a}\right)=\left(g_{3}^{a}, g_{1}^{a} g_{2}^{\theta_{a}}\right)$, and sends $\left(P_{a}, Q_{a}\right)$ to Bob. Bob does the symmetric version, computing and sending $\left(P_{b}, Q_{b}\right)=\left(g_{3}^{b}, g_{1}^{b} g_{2}^{\theta_{b}}\right)$, where $b \in_{R} \mathbb{Z}_{q}$. Alice calculates $R_{a}=\left(Q_{a} / Q_{b}\right)^{x_{a}}$. Bob also calculates $R_{b}=\left(Q_{a} / Q_{b}\right)^{x_{b}}$. Then, Alice sends $R_{a}$ with a proof that $\log _{g_{1}} g_{a}=\log _{Q_{a} / Q_{b}} R_{a}$ to Bob [17]. 
- Bob can now learn the result of the equality test by checking whether $P_{a} / P_{b}=R_{a}^{x_{b}}$. Then, he sends same proof as above to show $\log _{g_{1}} g_{b}=\log _{Q_{a} / Q_{b}} R_{b}$ so that Alice also learns the equality test result.

Note that if Bob does not send the last message, he will not obtain the equality signature $s_{e q}$ in our protocol, and the whole protocol will be aborted without anyone learning the actual output.

\section{B Making a Secure Protocol Fair}

Alice and Bob will evaluate a function $f(x, y)=\left(f_{a}(x, y), f_{b}(x, y)\right)$, where Alice has input $x$ and gets output $f_{a}(x, y)$, and Bob has input $y$ and gets output $f_{b}(x, y)$, and for simplicity of the presentation we have $f:\{0,1\}^{l} \times\{0,1\}^{l} \rightarrow\{0,1\}^{l} \times\{0,1\}^{l}$, where $l$ is an integer.

All commitments are Fujisaki-Okamoto commitments [25, 22] unless specified otherwise, and all encryptions are simplified Camenish-Shoup (sCS) encryptions as in [38]. We use the protocol [38] to adapt our fairness principles in Section 4. We do not explain all the steps that are exactly the same as in Section 4.

Alice and Bob agree on the circuit that computes $f(x, y)$. Then, they begin to construct their garbled circuits separately. The construction is quite similar for Alice and Bob. Therefore, we give details of the construction through Alice. When there is an important difference, we also show Bob's way of doing it.

Preparation Phase: It is the same explanation as in the Section 4.

S2PC Phase:

1. Commitments for keys: Alice generates keys $\left\{k_{w_{z}, t}^{A}\right\}_{z \in\{a, b\}, t \in\{0,1\}, w \in W \backslash W_{O}}$ representing left and right and 0 and 1 for each wire except the output gates' output wires. Then, she computes sCS commitments $\left\{C_{w_{z}, t}^{A}\right\}_{z \in\{a, b\}, t \in\{0,1\}, w \in W \backslash W_{O}}$ for each key as in [38].

Normal Gates: As in the Yao's protocol [64], she begins to construct the garbled tables. First, she picks permutation-pairwise bits $\varphi_{a}^{A}$ and $\varphi_{b}^{A}$ for each gate to permute the garbled table. Then she prepares the double encryptions of the keys according to permutation order (see Table 4).

Input Gates and Output Gates: It is contructed same way as in Section 4 and the encryption is done with using simplified Camenish-Shoup (sCS) [38].

See Table 4 for construction details of output and input gates.

\begin{tabular}{|c|c|c|c|c|}
\hline Row & $t_{a}, t_{b}$ & Output Bit $(t)$ & Garbled Input Gate & Garbled $O$ \\
\hline 00 & $\left(0 \oplus \varphi_{a}^{A}\right),(0 \oplus \varphi$ & $\left(t_{a} \vee t_{b}\right)$ & $E_{k_{w_{a, t a}}^{A}} E_{k_{w_{b, t_{b}}}^{A}}\left(k_{w_{o, t}}^{A}, s_{w_{b}^{g}, t}^{g}\right.$ & $E_{k_{w_{a, t_{a}}}^{A}} E_{k_{w_{b}}^{A},}$ \\
\hline 01 & (c) & & $E_{k_{w_{a, t_{a}}}^{A}} E_{k_{w_{b, t_{b}}}^{A}}^{A}\left(k_{w_{o, t}}^{A}, s_{w_{b}^{g}, t}\right)$ & $E_{k_{w_{a, t_{a}}}^{A}} E_{k_{w_{b, t_{b}}}^{A}}^{A}\left(\delta_{i+1}^{A}, \varepsilon_{i+1}^{A}\right.$ \\
\hline 10 & $\left(1, A^{A}\right)\left(0, A^{A}\right)$ & & $E_{k_{w_{a, t_{a}}}^{A}}^{A} E_{k_{w_{b, t_{b}}}^{A}}^{A}\left(k_{w_{o, t}}^{A}\right.$ & $E_{k_{w_{a, t_{a}}}^{A}} E_{k_{w_{b, t_{b}}}^{A}}$ \\
\hline 11 & 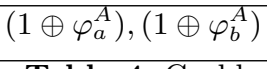 & & $k_{w_{w_{a}, t_{a}}^{A}} E_{k_{w_{b, t_{b}}}^{A}}\left(k_{w_{o}}^{A}\right.$ & $E_{k_{w_{a, t_{a}}}^{A} I}$ \\
\hline
\end{tabular}

Table 4. Garbled Input and Output Gates for an OR gate constructed by Alice.

2. They exchage garbled tables along with the commitmetments as in the Figure 1.

Step 3 an 4 are the same as in the explanation in Section 4.

Equality Phase:

Fair Exchange Phase: 